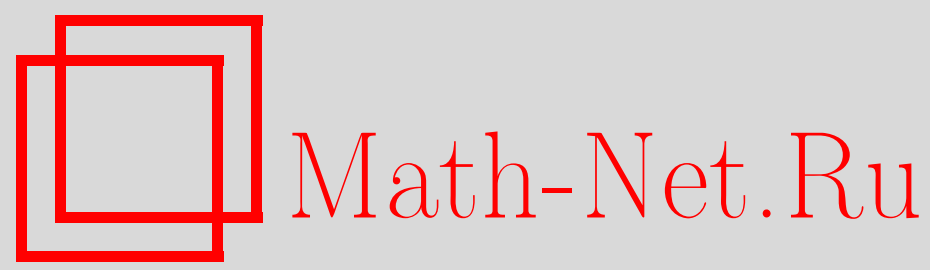

Н. Г. Мощевитин, О сублакунарных последовательностях и выигрышных множествах, Матем. заметки, 2005, том 78, выпуск 4, 634-637

DOI: https://doi.org/10.4213/mzm2626

Использование Общероссийского математического портала Math-Net.Ru подразумевает, что вы прочитали и согласны с пользовательским соглашением http://www . mathnet.ru/rus/agreement

Параметры загрузки:

IP: 35.174 .16 .151

26 апреля 2023 г., $15: 21: 16$

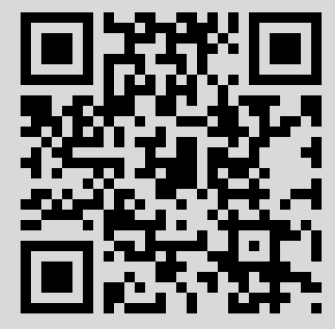




\section{О СУБЛАКУНАРНЫХ ПОСЛЕДОВАТЕЛЬНОСТЯХ И ВЫИГРЫШНЫХ МНОЖЕСТВАХ}

\section{Н. Г. Мощевитин}

Напомним определение $(\alpha, \beta)$ - игры Банаха-Мазура-Шмидта на вещественной прямой, $(\alpha, \beta)$ и $\alpha$-выигрышного множества и их простейшие свойства (см. [1], [2]). Пусть $0<\alpha, \beta<1$ и $S \subseteq \mathbb{R}$. Рассматривается игра, в которую играют черные и белые. Сначала черные выбирают на действительной оси замкнутый отрезок $B_{1}$ длины $l\left(B_{1}\right)=2 \rho$. Затем белые выбирают замкнутый отрезок $W_{1} \subset B_{1}$ длины $l\left(W_{1}\right)=\alpha l\left(B_{1}\right)$. Потом черные выбирают замкнутый отрезок $B_{2} \subset W_{1}$ длины $l\left(B_{2}\right)=\beta l\left(W_{1}\right)$, и т.д. В результате образуется последовательность вложенных замкнутых отрезков $B_{1} \supset W_{1} \supset B_{2} \supset W_{2} \supset \cdots$ с длинами

$$
l\left(B_{i}\right)=2(\alpha \beta)^{i-1} \rho \text { и } l\left(W_{i}\right)=2 \alpha(\alpha \beta)^{i-1} \rho, \quad i=1,2, \ldots .
$$

Очевидно, что множество $\bigcap_{i=1}^{\infty} B_{i}=\bigcap_{i=1}^{\infty} W_{i}$ состоит из одной единственной точки. Если $\bigcap_{i=1}^{\infty} B_{i} \in S$, то говорят, что белые выпграли игру. Множество $S$ называется $(\alpha, \beta)$-выигрышнblм, если белые могут выиграть игру независимо от того, как играют черные. Множество $S$ назьвается $\alpha$-выигрышным, если оно является $(\alpha, \beta)$-выигрышным при каждом $\beta \in(0,1)$.

Хорошо известно, что всякое $\alpha$-выпгрыштое множество на вещественной прямой при $\alpha>0$ имеет размерность Хаусдорфа, равную единице. Шмидт [1], [2] доказал, что пересечение любого счетного числа $\alpha$-выигрышных множеств снова будет $\alpha$-вьпгрышным. В качестве одного из примеров $\alpha$-выпгрышного множества с произвольным $\alpha \in(0,1 / 2)$ Шмидт рассматривал множество

$$
\mathscr{N}_{q}=\left\{x \in \mathbb{R}: \exists C(x)>0 \forall n \in \mathbb{N}\left\|q^{n} x\right\| \geqslant C(x)\right\},
$$

состоящее из чисел, не являющихся нормальными по натуральному основанию $q \geqslant 2$.

С другой стороны, Поллингтон [3] и де Матан [4], отвечая на вопрос Эрдеша [5], доказали, что для всякой лакунарной последовательности $T=\left\{t_{n}\right\}_{n+1}^{\infty}$ (т.е. для последовательности положительных чисел, удовлетворяющей условию

$$
\frac{t_{n+1}}{t_{n}} \geqslant \lambda \quad \forall n \in \mathbb{N}
$$

с некоторым $\lambda>1)$ множество

$$
\mathscr{N}(T)=\left\{x \in \mathbb{R}: \exists C(x)>0 \forall n \in \mathbb{N}\left\|t_{n} x\right\| \geqslant C(x)\right\}
$$

имеет хаусдорфову размерность единица (естественно, мера Лебега этого множества равна нулю). Легко проверить, что с точки зрения $(\alpha, \beta)$-игры множество $\mathcal{N}(T)$ будет $\alpha$-выигрышньп с любым $\alpha \in(0,1 / 2)$. В недавней работе Мощевитина и Ахунжанова [6] был получен ряд обобщений и усилений упомянутых результатов Поллингтона-де Матана. В частности, там было доказано, что для последовательности $T^{\prime}=\left\{t_{n}^{\prime}\right\}_{n+1}^{\infty}$, удовлетворяющей условию

$$
\frac{t_{n+1}^{\prime}}{t_{n}^{\prime}} \geqslant 1+\frac{1}{n^{\beta}} \quad \forall n \in \mathbb{N}
$$

при некотором $\beta \in(0,1 / 2]$ множество

$$
\mathscr{N}\left(T^{\prime}\right)=\left\{x \in \mathbb{R}: \exists C(x)>0 \forall n \in \mathbb{N}\left\|t_{n}^{\prime} x\right\| \geqslant \frac{C(x)}{n^{2 \beta}}\right\}
$$

имеет хаусдорфову размерность единица.

Автору неизвестен ответ на следующий вопрос: верно ли, что для любой последовательности $T^{\prime}$, удовлетворяющей условию (1), множество $\mathcal{N}\left(T^{\prime}\right)$ является $\alpha$-вынгрышным. Тем не менее, для некоторых последовательностей сублакунарного роста подобный результат получить несложно.

Работа вьполнена при поддержке гранта Президента РФ, грант № МД-3321.2004.1, программы "Ведущие научные школы”, грант № HШ-136.2003.1, и фонда INTAS, грант № 03-51-5070. 
Теорема. Пусть последовательность положительных чисел $t_{n}$ такова, что

$$
\forall \varepsilon>0 \quad \exists N_{0} \quad \forall n \geqslant N_{0}: \quad \frac{t_{n+1}}{t_{n}} \geqslant 1+\frac{1}{n^{\varepsilon}} .
$$

Тогда при кахдом положительном $\delta$ множество

$$
\mathscr{A}_{\delta}=\left\{x \in \mathbb{R}: \exists c(x)>0 \forall n \in \mathbb{N}\left\|t_{n} x\right\|>\frac{c(x)}{n^{\delta}}\right\}
$$

будет $\alpha$-выигрышным с любым заданным наперед $\alpha \in(0,1 / 2)$, m.е. $\operatorname{win} \operatorname{dim} \mathscr{A}_{\delta}=1 / 2$.

СледствиЕ. Пусть для последовательности положительных чисел $t_{n}$ выполнено (2). Тогда множество

$$
\mathscr{A}=\bigcap_{\delta>0} \mathscr{A}_{\delta}=\left\{x \in \mathbb{R}: \forall \delta>0 \exists c(x, \delta)>0 \forall n \in \mathbb{N}\left\|t_{n} x\right\|>\frac{c(x, \delta)}{n^{\delta}}\right\}
$$

будет $\alpha$-выигрышным с любым заданным наперед $\alpha \in(0,1 / 2)$, m.е. $\operatorname{win} \operatorname{dim} \mathscr{A}=1 / 2$.

ДОКАЗАТЕЛЬСТВО ТЕОРЕМЫ представляет собой модификацию оригинальной конструкции Шмидта [1], [2] с использованием соображений работ [3], [4] и развивающей их работы [6].

Зафиксируем $\alpha, \beta \in(0,1)$ такие, что $\gamma=\gamma(\alpha, \beta)=1+\alpha \beta-2 \alpha>0$. Положим

$$
d=d(\alpha, \beta)=\left\lceil\frac{\log _{2}(2 / \gamma)}{\log _{2}(1 /(\alpha \beta))}\right\rceil, \quad \tau=8 d \log _{2} \frac{1}{\alpha \beta}, \quad \varepsilon=\frac{\delta}{40 d \log _{2}(1 /(\alpha \beta))} .
$$

Будем считать, что при $n \geqslant N_{0}(\alpha, \beta, \delta)$ для рассматриваемой последовательности вьполнено $t_{n+1} / t_{n} \geqslant 1+1 / n^{\varepsilon}$ с $\varepsilon$, определенным в (3).

Определим рекуррентньм образом величины $n_{r}, k_{r}, \kappa_{r} \in \mathbb{N}$ из условий

$$
\begin{gathered}
k_{0}=n_{0}=1, \quad n_{r+1}=n_{r}+k_{r+1}, \quad \kappa_{r}=\left\lceil\log _{2} 4 k_{r}\right\rceil \geqslant 2, \\
k_{r+1}=\min \left\{k \in \mathbb{N}: \frac{t_{n_{r}+k}}{t_{n_{r}}} \geqslant\left(16 k_{r} k\right)^{\tau}\right\} .
\end{gathered}
$$

Сразу заметим, что $k_{r} \leqslant n_{r}$. Заметим также, что если положить $k^{*}=\left[n_{r}^{2 \varepsilon}\right]$, то

$$
\begin{aligned}
\frac{t_{n_{r}+k^{*}}}{t_{n_{r}}} & \geqslant \prod_{n=n_{r}}^{n_{r}+k^{*}-1}\left(1+\frac{1}{n^{\varepsilon}}\right) \geqslant \exp \left(\frac{1}{2} \sum_{n=n_{r}}^{n_{r}+k^{*}-1} \frac{1}{n^{\varepsilon}}\right) \geqslant \exp \left(\frac{1}{2} \int_{n_{r}}^{n_{r}+k^{*}} \frac{d x}{x^{\varepsilon}}\right) \\
& \geqslant \exp \left(\frac{1}{1(1-\varepsilon)}\left(\left(n_{r}+k^{*}\right)^{1-\varepsilon}-n_{r}^{1-\varepsilon}\right)\right) \geqslant \exp \left(\frac{k^{*}}{8 n^{\varepsilon}}\right) \\
& =\exp \left(\frac{n^{\varepsilon}}{8}\right) \geqslant\left(16 n_{r}\right)^{\tau(1+2 \varepsilon)} \geqslant\left(16 k_{r} k^{*}\right)^{\tau}
\end{aligned}
$$

при $n_{r} \geqslant N_{1}(\alpha, \beta, \delta)$. Следовательно, при $n_{r} \geqslant N_{2}(\alpha, \beta, \delta)=\max \left(N_{0}(\alpha, \beta, \delta), N_{1}(\alpha, \beta, \delta)\right)$ выполняется

$$
k_{r+1} \leqslant k^{*} \leqslant n_{r}^{2 \varepsilon}, \quad \kappa_{r+1} \leqslant 2 \varepsilon \log _{2} n_{r}+3 .
$$

Из монотонности последовательности $n_{r}$ видим, что

$$
k_{r} \leqslant n_{r}^{2 \varepsilon}, \quad \kappa_{r} \leqslant 2 \varepsilon \log _{2} n_{r}
$$

Определим величины

$$
\Delta_{r}=\min \left\{\frac{1}{t_{n_{r+1}-1}} ; \frac{(\alpha \beta)^{1+2 d \kappa_{r}}}{t_{n_{r}-1}}\right\}
$$


Из определения $\Delta_{r}$ очевидно, что

$$
\Delta_{r} \geqslant \frac{(\alpha \beta)^{1+2 d \kappa_{r}}}{t_{n_{r+1}-1}}
$$

Докажем неравенство

$$
\frac{\Delta_{r}}{\Delta_{r+1}} \geqslant\left(\frac{1}{\alpha \beta}\right)^{1+2 d \kappa_{r+1}}
$$

Действительно, в случае, когда $\Delta_{r}=1 / t_{n_{r+1}-1}$, используя неравенство

$$
\Delta_{r+1} \leqslant \frac{(\alpha \beta)^{1+2 d \kappa_{r+1}}}{t_{n_{r+1}-1}}
$$

сразу получаем (7). Если же $\Delta_{r}=(\alpha \beta)^{1+2 d \kappa_{r}} / t_{n_{r}-1}$, то используем неравенство $\Delta_{r+1} \leqslant$ $1 / t_{n_{r+2}-1}$ и оценку $t_{n_{r+2}-1} / t_{n_{r}-1} \geqslant t_{n_{r+1}} / t_{n_{r}} \geqslant\left(16 k_{r} k_{r+1}\right)^{\tau}$ :

$$
\begin{aligned}
\frac{\Delta_{r}}{\Delta_{r+1}} & \geqslant \frac{t_{n_{r+2}-1}}{t_{n_{r}-1}}(\alpha \beta)^{1+2 d \kappa_{r}} \geqslant\left(16 k_{r} k_{r+1}\right)^{\tau}(\alpha \beta)^{1+2 d \kappa_{r}} \\
& \geqslant\left(\frac{1}{\alpha \beta}\right)^{\tau\left(\log _{2} 4 k_{r}+\log _{2} 4 k_{r+1}\right) / \log (1 /(\alpha \beta))-1-2 d \kappa_{r}} .
\end{aligned}
$$

Здесь показатель в последнем выражении оценивается снизу через

$$
\frac{\tau}{\log (1 /(\alpha \beta))}\left(\kappa_{r}+\kappa_{r+1}-2\right)-1-2 d \kappa_{r} \geqslant 1+2 \kappa_{r+1}
$$

в силу выбора параметра $\tau$ по формуле из (3). Из последних двух оценок соотношение (7) следует и в этом случае.

Теперь будем непосредственно доказывать $(\alpha, \beta)$-вьнирышность множества $\mathscr{A}_{\delta}$. Пусть черные начали $(\alpha, \beta)$-игру с отрезка $B_{1}$ радиуса $\rho$. Возьмем достаточно болшое $j$, чтобы $n_{j} \geqslant N_{2}$ и, кроме того, $\Delta_{j}<\rho$, и определим натуральные величины $m_{r}$ из условий

$$
\Delta_{r+j}>2 \rho(\alpha \beta)^{m_{r}-1} \geqslant \alpha \beta \Delta_{r+j} .
$$

Отрезок $B_{m_{r}}$ содержит в себе не более $k_{r+j+1}$ рациональных чисел вида $a / t_{n}, a \in \mathbb{Z}, n_{r+j} \leqslant$ $n<n_{r+j+1}$. В [2] (см. лемма 1В на с. 44) доказано, что если в $(\alpha, \beta)$-игре встретился черный отрезок $B_{q}$ с центром $b$, то белые могут играть так, что (не зависимо от того, как играют черные) отрезок $W_{q+d}$ будет лежать по заданную сторону от точки $b$ и, кроме того, расстояние от отрезка $W_{q+d}$ до точки $b$ будет $\geqslant \gamma\left|B_{q}\right| / 4$. За $d \kappa_{r+j+1}$ шагов игры белые, применяя эту лемму Шмидта и процесс дихотомии, могут добиться того, чтобы отрезок $B_{m_{r}}+d \kappa_{r+j+1}$ не только не содержал отмеченные рациональные числа, но и было бы вьполнено

$$
x \in B_{m_{r}+d \kappa_{r+j+1}} \Longrightarrow\left|x-\frac{a}{t_{n}}\right| \geqslant \frac{\gamma\left|B_{m_{r}+d\left(\kappa_{r+j+1}-1\right)}\right|}{4}, \quad a \in \mathbb{Z}, \quad n_{r+j} \leqslant n<n_{r+j+1} .
$$

Это значит, что для $x \in B_{m_{r}+d \kappa_{r+j+1}}$ для $n_{r+j} \leqslant n<n_{r+j+1}$ выполняется

$$
\left|x-\frac{a}{t_{n}}\right|>\frac{\gamma \rho}{2} \cdot(\alpha \beta)^{m_{r}-1} \cdot(\alpha \beta)^{d \kappa_{r+j+1}-1} \geqslant \frac{\gamma}{4} \Delta_{r+j}(\alpha \beta)^{d \kappa_{r+j+1}} \geqslant \frac{\alpha \beta \gamma}{4} \cdot \frac{(\alpha \beta)^{2 d\left(\kappa_{r+j}+\kappa_{r+j+1}\right)}}{t_{n_{r+j+1}-1}}
$$

(в последней вык ладке использована нижняя оценка из (8) и неравенство (6)). Таким образом, при $n_{r+j} \leqslant n<n_{r+j+1}$ имеем

$$
\left\|t_{n} x\right\| \geqslant \frac{\alpha \beta \gamma \rho}{2}(\alpha \beta)^{2 d\left(\kappa_{r+j}+\kappa_{r+j+1}\right)} \frac{t_{n_{r+j}}}{t_{n_{r+j+1}-1}} .
$$


Но из определения величины $n_{r+j+1}$ следует, что

$$
\frac{t_{n_{r+j}}}{t_{n_{r+j+1}-1}} \geqslant\left(16 k_{r+j} k_{r+j+1}\right)^{-\tau}
$$

Теперь, в силу выбора параметров $\varepsilon, \tau$ по формулам из (3) и в силу (4), (5) получаем

$$
\left\|t_{n} x\right\| \geqslant \frac{\alpha \beta \gamma}{4} \cdot \frac{(\alpha \beta)^{2 d\left(\kappa_{r+j}+\kappa_{r+j+1}\right)}}{\left(16 k_{r+j} k_{r+j+1}\right)^{\tau}} \geqslant \frac{C(\alpha, \beta)}{n^{\delta}}, \quad C(\alpha, \beta)>0, \quad n_{r+j} \leqslant n<n_{r+j+1} .
$$

Таким образом, если оказалось, что

$$
m_{r+1} \geqslant m_{r}+d \kappa_{r+j+1}
$$

то мы построили индуктивный процесс, обеспечивающий для белых в $(\alpha, \beta)$-игре возможность добиться того, чтобы для $x=\bigcap_{m} B_{m}$ было бы выполнено

$$
\left\|t_{n} x\right\| \geqslant \frac{C(\alpha, \beta)}{n^{\delta}} \quad \forall n>n_{j+1} .
$$

Убедимся, что неравенство (9) справедливо. Действительно из (8) получаем

$$
(\alpha \beta)^{m_{r+1}-m_{r}} \leqslant \frac{2 \rho(\alpha \beta)^{m_{r+1}-1}}{2 \rho(\alpha \beta)^{m_{r}-1}} \leqslant \frac{\Delta_{r+j+1}}{\alpha \beta \Delta_{r+j}} .
$$

Подставим сюда неравенство (7) с заменой $r$ на $r+j$ и убедимся, что

$$
m_{r+1}-m_{r} \geqslant 2 d \kappa_{r+j+1}>d \kappa_{r+j+1} .
$$

Итак, мы показали, что в $(\alpha, \beta)$-игре белые могут добиться от точки $x=\bigcap_{m} B_{m}$ выполнения условия (10). Чтобы неравенство из (10) вьполнялось не только для $n>n_{j+1}$, но и для всех натуральных $n$ (возможно, с заменой константы $C(\alpha, \beta)$ на меньшую величину $C^{\prime}(\alpha, \beta, \rho, \delta)$ ), нам надо проследить за конечным (зависящим от $\alpha, \beta, \delta, \rho$ ) количеством точек вида

$$
\frac{a}{t_{n}}, \quad a \in \mathbb{Z}, \quad n \leqslant n_{j+1},
$$

попавших в самом начале игры в отрезок $B_{1}$. Но, если сравнить (9) и (11), то видно, что на каждом промежутке игры между моментами $m_{r}$ и $m_{r+1}$ остается запас в $d \kappa_{i}$ шагов, который, в частности, позволяет оторваться от любого конечного набора точек и обеспечить для точки $x=\bigcup_{m} B_{m}$ неравенство

$$
\left\|t_{n} x\right\| \geqslant \psi_{n} \quad \forall n \leqslant n_{j+1}
$$

с некоторыми положительными $\psi_{n}$, зависящими от $\alpha, \beta, \delta$.

Теорема доказана.

\section{СПИСОК ЦИТИРОВАННОЙ ЛИТЕРАТУРЫ}

1. Schmidt W. M. // Trans. Amer. Math. Soc. 1966. V. 623. P. 178-199. 2. Шмидт В. М. Диофантовы приближения. М.: Мир, 1983. 3. Pollington A. D. // Illinois J. Math. 1979. V. 23. P. 511-702. 4. de Mathan D. // Acta Math. Hungar. 1980. V. 36. P. 237-241. 5. Erdös P. Repartition Modulo 1. Lecture Notes in Math. V. 475. New York: Springer-Verlag, 1975. 6. Ахунжанов Р. К., Мощевитин Н. Г. // Матем. заметки. 2005. Т. 77. № 6. С. 803-813. 